\title{
Consideraciones clínicas en el manejo de las reabsorciones inflamatorias dentinarias internas
}

Clinical considerations in the management of internal inflammatory dentin resorption
Considerações clinicas no manejo da reabsorção inflamatória interna dentinária
Fecha de Recepción

21 de diciembre 2018
Aceptado para su publicación

20 de marzo 2019
Natalia Belén Montiel

Asignatura Endodoncia. Módulos Clínica Rehabilitadora I y II. Facultad de Odontología.

UNNE

Mariel Beatriz Galiana

Asignatura Endodoncia. Módulos Clínica Rehabilitadora I y II. Facultad de Odontología. UNNE.

Carlos Daniel Lugo De Langhe. Asignatura Endodoncia. Módulos Clínica Rehabilitadora I y II. Facultad de Odontología. UNNE.

Graciela Mónica Gualdoni.

Asignatura Endodoncia. Módulos Clínica Rehabilitadora / y II. Facultad de Odontología. UNNE.

\section{Autor de Correspondencia:}

Natalia Belén Montiel

Facultad de Odontología. UNNE. Av. Libertad 5450. Corrientes (Capital).

República Argentina.

Email: natybelenmontiel@hotmail.com

\section{Resumen}

Las reabsorciones dentinarias internas son procesos patológicos que afectan a las piezas dentarias y comprometen su estabilidad en boca si no se las trata a tiempo. El diagnóstico precoz es de fundamental importancia porque evitaría la futura pérdida de la pieza dentaria afectada, sin embargo, existen situaciones donde su hallazgo se realiza de manera ocasional ante una toma radiográfica de rutina o ante la presencia de alguna coloración rosada a nivel de la corona dentaria lo cual manifiesta que la lesión compromete la cámara pulpar. Su hallazgo implica un desafío para el endodoncista que deberá intervenir en forma inmediata ante su detección. Es por ello que el artículo a través de la búsqueda bibliográfica en diferentes bases de datos analiza la información acerca de los aspectos clínicos de diagnóstico temprano y su intervención detallando aspectos de interés para el clínico que se enfrente ante esta patología, destacando la importancia de realizar el tratamiento endodóntico en forma inmediata y eliminar el tejido pulpar inflamatorio que es la causa principal del proceso reabsortivo. Instaurada la terapéutica endodóntica se realizará la restauración definitiva de la pieza dentaria y los controles posoperatorios en el tiempo.

Palabras claves: Resorción Dentaria, Osteoclastos, Cavidad Pulpar. 


\section{Abstract}

Internal dentin resorptions are pathological processes that affect the dental pieces and compromise their stability in the mouth if they are not treated on time. Early diagnosis is of fundamental importance because it would prevent the future loss of the affected tooth, however, there are situations where the finding is occasionally made when taking a routine $X$-ray or in the presence of some pink coloration at the level of the dental crown, which shows that the lesion compromises the pulp chamber. Its finding implies a challenge to the endodontist who must intervene immediately as soon as it is detected. By means of bibliographic research in different databases, this work analyzes the information about the clinical aspects of early diagnosis and its intervention. It details aspects of interest for the clinician who faces this pathology. It highlights the importance of performing the endodontic treatment immediately and eliminating the inflammatory pulp tissue that is the main cause of the reabsorptive process. Once the endodontic treatment is established, the final restoration of the tooth will take place and the postoperative controls will be carried out over time.

Key Words: Tooth Resorption, Osteoclast, Dental Pulp Cavity

\section{Resumo}

Reabsorção interna da dentina é um processo patológico que afeta às peças dentárias e compromete a estabilidade das mesmas na boca se não forem tratadas a tempo. $O$ diagnóstico precoce é de fundamental importância, pois evitaria a perda futura da peça dentária afetada, no entanto, existem situações em que o achado é feito ocasionalmente na presença de uma radiografia de rotina ou na presença de alguma coloração rosa na coroa do dente, o que mostra que a lesão compromete a câmara pulpar. Sua constatação implica um desafio para endodontista que deve intervir imediatamente depois da detecção. É por isso que o artigo, através da busca bibliográfica em diferentes bases de dados, analisa as informações sobre os aspectos clínicos do diagnóstico precoce e sua intervenção detalhando aspectos de interesse para o clínico que enfrenta esta patologia destacando a importância da realização do tratamento endodôntico imediatamente e eliminando o tecido pulpar inflamatório que é a principal causa do processo reabsortivo. Uma vez que o tratamento endodôntico tenha sido estabelecido, a restauração final do dente e os controles pós-operatórios serão realizados ao longo do tempo.

Palavras chaves: Reabsorção de Dente, Osteoclastos, Cavidade Pulpar

\section{Introducción}

Las reabsorciones dentarias son procesos fisiológicos o patológicos que afectan a los tejidos mineralizados de las piezas dentarias ${ }^{1-6}$ e involucran una compleja interacción entre células inflamatorias, células que producen reabsorción y células formativas asociadas a la matriz extracelular ${ }^{7}$.

Las reabsorciones fisiológicas se producen en las piezas deciduas durante la exfoliación de las mismas y las patológicas se producen en piezas dentarias permanentes ${ }^{1,2,7,8,9}$.

La resorción dentaria interna (RDI) es también conocida como Hiperplasia Crónica Perforante de la Pulpa, Granuloma Interno, Diente Rosado de Mummery 24,7,9,10,11. Es una patología que causa la disolución patológica de las estructuras dentales mineralizadas tales como la dentina $\circ$ el cemento, ${ }^{12,13}$ debido a la actividad de células odontoclásticas ${ }^{5,14,15}$.

Es una rara condición que dificulta el tratamiento endodóntico ya que en su evolución involucra tejidos mineralizados como dentina, cemento, hueso y estructuras no mineralizadas que permiten un intercambio biológico cuyo desequilibrio desencadena una patología. Es de importancia establecer el tratamiento inmediato ante la detección de esta patología ya que en su evolución puede abarcar mayor cantidad de tejido mineralizado y llegar a comprometer la permanencia de la pieza dentaria en boca.

Las causas que la producen son de diferente naturaleza, como lesiones e irritaciones del ligamento periodontal y/o de la pulpa dental, como secuela de un traumatismo dentario, ${ }^{2,16}$ luxación traumática, por movimientos orto- 
REVISTA FACULTAD DE ODONTOLOGÍA

ISSN 1668-7280 / ISSN-E 2683-7986

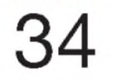

dónticos, ${ }^{6}$ infecciones pulpares crónicas o de las estructuras periodontales $5,7,10,17,18,19$.

Otros factores asociados a su origen pueden ser trastornos hormonales como el hipertiroidismo que puede mostrar más de una pieza dentaria afectada por el proceso. Así también las maniobras operatorias efectuadas por el clínico odontólogo, como la escasa refrigeración de las cavidades operatorias, inadecuada protección pulpodentinaria, el pulido excesivo de restauraciones pueden desencadenar el inicio de la patología. Al indagar sobre las posibles causas de su origen también se debería considerar las ocasiones en que ocurre esta patología en forma idiopática; sin causa aparente ${ }^{20}$.

La reabsorción inflamatoria interna se inicia luego de la lesión de la capa odontoblástica y de la predentina $2,3,9,19,21$.

Diferentes factores como traumas, agentes químicos, físicos ${ }^{13}$ agresión de agentes biológicos pueden desorganizar la capa odontoblástica y causar daño a la predentina ${ }^{3}$ creando así las condiciones iniciales necesarias para empezar el proceso de reabsorción. Este tipo de reabsorción es autolimitante y será transitoria ${ }^{12}$ mientras no existan factores estimulantes que se mantengan en el tiempo. Ante un estimulo duradero (inflamación) la reabsorción podrá progresar $^{12}$ hasta alcanzar el ligamento periodontal estableciéndose una comunicación entre el interior $y$ el exterior del diente ${ }^{7}$, pasando a ser reabsorciones comunicantes'.

Cuando comienza el proceso de reabsorción el tejido pulpar es vital; aunque en general presenta una inflamación crónica de larga data. Existen situaciones en la que ese proceso inflamatorio crea las condiciones para que la supervivencia del tejido pulpar se vea comprometida y comience el proceso de mortificación pulpar. La mortificación pulpar parcial genera productos que estimulan el proceso de reabsorción. Así mismo la presencia de microorganismos instalados desde el primer momento que comienza el proceso inflamatorio o luego del inicio de la mortificación pulpar son los factores responsables de la continuidad del proceso destructivo.

Una mortificación pulpar total y la falta de condiciones para la supervivencia de las células responsables de la reabsorción hacen que esta se detenga. Es decir que la condición indispen- sable para que se produzca la misma es la vitalidad del tejido pulpar, ${ }^{4}$.

Aunque el mecanismo inmunohistoquimico del proceso de reabsorción no se conoce con exactitud; ciertos componentes como osteoprotegerina, el activador del receptor de ligando del factor nuclear kappa-B (RANKL), el activador del receptor del factor nuclear kappa-B (RANK) y el factor estimulante de colonias de macrófagos (MCSF) se consideran importantes para la cascada inflamatoria dentro del tejido pulpar que dispara la diferenciación de células madre a odontoclastos o macrófagos para desarrollar dentina ${ }^{22}$.

Las lesiones o irritaciones de los tejidos duros provocan una serie de cambios químicos que dan como resultado la formación de células gigantes multinucleadas que se denominan clastos, que son las responsables de los procesos de reabsorción y se acompañan de macrófagos y monocitos organizando una compleja interacción de eventos biológicos moleculares que involucran citoquinas, enzimas y hormonas, que influyen en la progresión de la patología ${ }^{23}$.

El objetivo del trabajo es realizar una revisión bibliográfica sobre los aspectos clínicos diagnósticos de esta patología y su posible tratamiento.

\section{Metodología}

Para ello se llevó a cabo una búsqueda bibliográfica en las base de datos Ebsco, Scielo y PubMed de los últimos 15 años. Se tuvieron en cuenta artículos que brindaron información exclusiva de reabsorciones dentinarias internas, que abarcaba casos clínicos y revisiones cuyos idiomas fueron español e inglés. Se utilizó para la búsqueda las siguientes palabras, cavidad pulpar, osteoclastos, reabsorciones dentarias. Con la información obtenida de los artículos analizados se buscaron los aspectos más destacados de su presentación en base a etiología, diagnóstico y tratamiento. 


\section{Diagnóstico}

La mayoría de las reabsorciones dentinarias internas no presentan signos ni síntomas clínicos tempranos ${ }^{2}$.

La identificación de este tipo de reabsorciones puede hacerse a través de un examen casual o intencional. Durante una visita de rutina o de control puede descubrirse por casualidad ya que generalmente el diente con reabsorción es asintomático $4,5,8,9,23$. Sin embargo, existen situaciones, en etapas avanzadas que podrán cursar con un cuadro clínico de dolor, hinchazón, decoloración de la pieza dentaria afectada ${ }^{22}$.

En los casos en que existe perforación de la raíz, la presencia de un trayecto fistuloso confirmaría la infección del conducto radicular ${ }^{2,19}$. En estos casos la corona de la pieza dentaria afectada podría mostrar una coloración grisácea².

Las reabsorciones internas confinadas a la cámara pulpar después de destruir la dentina pueden alcanzar el esmalte y mostrar un punto - mancha rosada que se transparenta a través de la superficie de esmalte ${ }^{19,22,24}$. Este cambio de color se debe a la invasión del tejido de granulación en la zona donde ha ocurrido la pérdida de tejido dentario, sustituyéndolo 4 .

Este signo que aparece en la porción coronaria se consideraría como patognomónico en los casos clínicos de reabsorción dentinaria interna $^{12,25}$.

El proceso de reabsorción es silencioso ${ }^{12}$ y el examen radiográfico es decisivo para su identificación, cabe destacar que éste solo demostrará las reabsorciones de mayor tamaño pudiendo esconder aquellas reabsorciones pequeñas o de corta data. La radiografía mostrará una imagen radiolúcida que altera la forma de la cavidad pulpar $^{25}$ semejante a una burbuja radiolúcida, con una forma oval, con características simétricas y densidad uniforme, ${ }^{23}$ que se continúa con el canal radicular distorcionándolo de su forma habitual," con un aumento de la luz del conducto con desaparición de las líneas que lo delimitan, distorsionando el contorno del conducto radicular ${ }^{22}$.

El examen intencional se hará en aquellas situaciones donde se realiza un control a distancia de piezas dentarias que hayan sufrido algún tipo de traumatismo ${ }^{13}$. Es importante destacar que las lesiones de reabsorción no aparecerán de manera inmediata, y las mismas se podrán iniciar a las $2 \circ 3$ semanas del traumatismo y ser evidenciadas en las radiografías entre los 60 y 90 días posteriores'. La radiografía convencional ofrece ciertas limitaciones ya que solo proporciona una imagen bidimensional dando cierta inexactitud en la imagen proporcionada en cuanto a su naturaleza y ubicación ${ }^{16,20}$.

El examen radiográfico no mostrará lesiones pequeñas ${ }^{18,22}$ ya que estas quedaran cubiertas por la dentina situada por delante o detrás del defecto. Es por ello que un examen radiográfico minucioso que muestre puntos diminutos de reabsorción es de interés para que se estudie la evolución y se apliquen técnicas radiográficas en diferentes angulaciones para entablar un tratamiento lo más pronto posible. La obtención de dos 0 tres radiografías en diferentes angulaciones puede ser de utilidad para demostrar el tipo de reabsorción dentinaria interna, la cual acompañará al conducto en todas las tomas'.

Otra de las herramientas diagnósticas en el campo de la endodoncia es la tomografía axial computarizada Cone Bean (CBCT) ${ }^{12}$ tecnología altamente útil e indispensable que permite una inspección 3D, en las vistas axial, sagital y coronal sin superposiciones anatómicas ${ }^{11,21,24}$ que aportará grandes beneficios por la exactitud de información en la detección de lesiones en estadios tempranos, ${ }^{17,26}$ permitirá detectar la extensión de las lesiones y/ o los sitios con las posibles comunicaciones con el ligamento periodontal|'16,20,24.

\section{Diagnóstico diferencial}

Es de importancia establecer un diagnóstico preciso de la patología ya que de ello dependerá la planificación del tratamiento a realizar. Las radiografías convencionales y la tomografía Cone Bean son los recursos diagnósticos más importantes que brindarán información sobre el tipo de reabsorción presente.

Los cambios en las angulaciones de las radiografías brindarán una información acerca de si el defecto de reabsorción es de tipo interno o externo ${ }^{10,27}$. Aplicando la regla mesial, bucal, distal (Ley del objeto bucal, MBD) la lesión externa se desviará de su posición y quedará superpuesta 
REVISTA FACULTAD DE ODONTOLOGÍA ISSN 1668-7280 / ISSN-E 2683-7986

36

sobre el sistema de conductos radiculares en la radiografía mesiorradial, pudiendo apreciarse radiolucideces tanto en la raíz como en el hueso adyacente ${ }^{10}$ cuando éste último está afectado. La RDI no se desplaza, ${ }^{20}$ es decir se observa cerca del conducto, presenta contornos definidos $y$ regulares ${ }^{20,23}$.

Un defecto localizado en la cara externa de la raíz se aleja del conducto a medida que cambia la angulación. ${ }^{(10)}$ Mediante la utilización de la ley del objeto bucal es posible distinguir si el defecto radicular externo es vestibular o palatolingual.

Las caries pueden ser fácilmente diferenciadas de la RDI por el hecho de que, al igual que en la reabsorción externa, se observa la configuración del conducto inalterada y se puede seguir a través de la lesión. El reborde de una lesión cariosa está menos definido.

Otra diferenciación debe hacerse con las reabsorciones cervicales externas, donde las radiografías muestran una línea opaca al lado del conducto radicular ${ }^{2}$.

Esta línea opaca es un signo diagnóstico diferencial fiable de la reabsorción de la raíz cervical. Las lesiones de reabsorción externa generalmente se presentan con bordes indefinidos y asimétricos con variaciones en la radiodensidad del cuerpo de la lesión ${ }^{11,20}$.

Las tomografías computarizadas facilitan enormemente el diagnóstico diferencial y ayudan a determinar las ubicaciones, dimensiones, propagación y posibles sitios de perforación en mayor detalle" lo que posibilita una mejor planificación, ${ }^{4,21,27}$ tratamiento y seguimiento ${ }^{20}$.

\section{Tratamiento}

El diagnóstico correcto del tipo de reabsorción ante el que se está presente es fundamental para instaurar el tratamiento específico de la misma. La selección del tratamiento adecuado en casos de resorción está relacionada con control de los factores de estimulación, es decir, control de la infección intrapulpal, ${ }^{20}$ extirpación del tejido de granulación y relleno del defecto de resorción ${ }^{28}$. Tan pronto se detecte la presencia de una reabsorción dentinaria interna la terapia endodóntica deberá ser instaurada para evitar el progreso de la pato- logía y que éste afecte la integridad de la pieza dentaria afectada ${ }^{23}$.

La conducta clínica a seguir es el tratamiento endodóntico convencional, ${ }^{7,29}$ eliminando el tejido pulpar inflamado $0^{4,8,27,30}$ que contiene las células responsables del proceso reabsortivo.

Es imprescindible tener presente ciertas premisas para el abordaje del conducto y eliminación completa del tejido pulpar. Se debe ampliar bien el segmento del conducto que va desde la cámara pulpar hasta el área reabsorbida. Se debe precurvar las limas para abordar el sitio de la reabsorción y una irrigación abundante con hipoclorito de sodio, ${ }^{3,17,18,20,23}$ al 2,5\% 1,19 o al $5,25 \%, 10$ para eliminar todo el tejido pulpar que contiene las células clásticas responsables del proceso reabsortivo. Así mismo la utilización de ultrasonido, ${ }^{2,4,7,18}$ ayudará a la mejor acción del hipoclorito de sodio, ${ }^{2}$ sobre todo en los sitios más inaccesibles, ${ }^{13}$ del defecto ${ }^{19}$.

La utilización de ultrasonido ${ }^{4}$ es de gran importancia ya que permite un mayor grado de limpieza ejerciendo una acción sinérgica de actividad tanto física como química. Las actividades físicas incluyen la cavitación y la corriente acústica. La cavitación se refiere al crecimiento y colapso de pequeñas burbujas en un medio fluido, causando la irradiación de ondas de choque que producen un muy efectivo mecanismo de limpieza en los espacios irregulares de los conductos ${ }^{7}$. La corriente acústica es el movimiento armónico del irrigante alrededor de la lima energetizada, ayudando a la remoción de los detritus. La actividad química está asociada con la activación ultrasónica del irrigante bactericida.

En ciertas ocasiones es recomendable una medicación intraconducto ${ }^{2,3,7,27,30}$ con pasta alcalina de hidróxido de calcio ${ }^{5,19,20,23}$ durante 4 semanas con dos o tres cambios para inhibir la actividad y fijación de células clásticas responsables del proceso de reabsorción. La aplicación del hidróxido de calcio coadyuvaría al colapso del tejido de granulación presente ${ }^{12,17,30}$ en las zonas más inaccesibles del defecto, ${ }^{(4)}$ debido a su efecto de disolución de la materia orgánica, efecto antibacteriano, antiflamatorio y estimulante de reparación de los tejidos ${ }^{3}$. Una vez limpio y conformado el conducto radicular se deberá obturar el mismo sellando el canal de manera tridimensional' ${ }^{13}$. Lo ideal es utilizar una 
técnica de gutapercha termoplastificada ${ }^{3,7,13,30,31}$ la cual permitirá un relleno adecuado en el sitio del defecto. Así mismo se puede usar una combinación de técnicas para rellenar las diferentes porciones del conducto ${ }^{2}$. En el tercio apical se puede utilizar la técnica de compactación lateral $y$ en el sitio del defecto una técnica de gutapercha termoplastificada ${ }^{4,13,32} \circ$ bien una técnica de gutapercha reblandecida con eucopercha. En tanto, se puede optar por la utilización de un sistema obturador con gutapercha flow, el cual es un sistema innovador de gutapercha en frío que combina dos productos en uno: gutapercha en polvo con un tamaño de partículas inferior a 30 um y un sellador. Este último sistema evolucionó en gutaflow ${ }^{2}$ que se presenta en una jeringa dispensadora de doble cuerpo que trae sus dos componentes separados y puntas de mezcla, esto disminuye el costo total del sistema de obturación.

El sitio del defecto deberá quedar obturado de la forma más correcta posible y posteriormente se procederá a la restauración específica de la pieza dentaria.

Actualmente las técnicas de tratamiento endodóntico regenerativo se presentan como otra alternativa de tratamiento para tratar estas situaciones de RDI. ${ }^{22}$ En los casos en que exista comunicación con el periodonto la opción quirúrgica $^{22}$ deberá implementarse pudiendo utilizarse el agregado de trióxido mineral (MTA) ${ }^{2}$, ${ }^{12,31}$ en el sitio del defecto $2,3,17,18,27-30$. El MTA es un material favorecedor de la reparación de dichas lesiones gracias a las propiedades de biocompatibilidad, buen sellado, bactericida, radiopacidad, osteogénico, cementogénico y regenerador de la nueva inserción del ligamento periodontal, lo cual aporta un beneficio en estos casos clínicos ${ }^{5,14}$. Asimismo existe actualmente otro material; el Biodentine, ${ }^{13}$ formado por silicato tricálcico, carbonato cálcico y óxido de zirconio, que es un sustituyente dentinario, cuya biocompatibilidad y respuesta biológica reparadora a nivel de la pulpay tejidos periapicales hacen de este material una alternativa en situaciones de perforaciones, apexificaciones $y$ relleno en el conducto radicular, favoreciendo un medio propicio para la reparación ${ }^{14}$. Por su capacidad osteogénica y cementogénica este material podría ser usado para rellenar el sitio del defecto junto con un adecuado sellado coronario favoreciendo el tratamiento de la reabsorción ${ }^{14}$. El relleno con Biodentine en el sitio del defecto puede proporcionar mayor resistencia reforzando las paredes del conducto que se encuentren debilitadas ${ }^{13,32}$.

Existen situaciones clínicas que provocan una comunicación externa con el tejido periodontal que requerirán de otras formas de abordaje clínico; como abordaje quirúrgico, eliminación de tejido de granulación circundante, aplicación de ácido tetraacético, agregado de relleno óseo y hasta membrana de contención para el mismo. Siempre considerando un conjunto de condicionantes que propicien este tipo de tratamiento, ya que el pronóstico en estos casos es muy reservado. ${ }^{28}$.

La indicación de extracción es imprescindible cuando el defecto haya debilitado mucho la pieza dentaria y su estabilidad en boca es comprometida ${ }^{2,22}$.

En casos en que la extracción dentaria sea necesaria, la alternativa de tratamiento implantológico es otra de las opciones a considerar; sobre todo en casos donde exista un compromiso estético y funcional. Sin embargo, el factor económico es uno de los condicionantes presentes a la hora de elección de este tratamiento ${ }^{15}$.

Todas las maniobras que puedan realizarse para el tratamiento endodontico se podrán complementar con el uso de magnificación como el uso de lupas o del microscopio para mejorar las maniobras del procedimiento ${ }^{19}$. Luego de la intervención realizada; los controles radiográficos serán indispensables en el tiempo para observar la evolución del caso.

\section{Conclusiones}

Debido al carácter asintomático de esta patología, en el diagnóstico precoz de las reabsorciones dentarias inflamatorias internas es fundamental la evidencia radiográfica y tomográfíca, que posibilitará el tratamiento inmediato evitando de esta manera la pérdida de las piezas dentarias afectadas por este proceso. La diversidad de posibilidades en el tratamiento que existen en la actualidad, como ser: los nuevos materiales incorporados al mercado, la intervención inme- 
REVISTA FACULTAD DE ODONTOLOGÍA

ISSN 1668-7280 / ISSN-E 2683-7986

38
DIVULGACIÓN

Montiel - Galiana - Lugo De Langhe - Gualdoni

http://dx.doi.org/10.30972/fo. 1213872

diata del tratamiento endodóntico y la obturación tridimensional del sistema de conductos radiculares, posibilitará una tasa de éxito elevada para la detención del proceso de reabsorción. Sin embargo, es imprescindible un seguimiento y control adecuado en el tiempo para indagar sobre aspectos de evolución favorable de esta patología.

\section{Referencias Bibliográficas}

I. Soares IJ, Goldberg F. Endodoncia. Técnica y fundamentos. 2 ed. Buenos Aires: Editorial Médica Panamericana; 2012.

2. Shenoi PR, Morey ES, Sonarkar SS, Kubde $R$, Badole GP, Singare P. Internal Root Resorption (Pink Tooth of Mummery) a Review. Int J Dent Clin. 20I5; 7 (I): 2530.

3. Andrade CF, Morais ND, Demenech LS, et al. Endodontic treatment of internal root resorption: a case report. RSBO. 2016; 13 (2): $|3|-\mid 37$.

4. Bains R, Tikku AP, Chandra A, Verma P. Internal Resorption: Clinical Perspective and Treatment Challenges. Asian J Oral Health Allied Sci. Jul 20I 5; 5 (2): 37-43.

5. Perocco AL. Tratamiento de la Reabsorcion dentinaria Interna. Mendoza, Noviembre 2017. Trabajo para optar al título de Especialista en Endodoncia. Disponible: http:// bdigital.uncu.edu.ar/objetos_digitales/9898/ perocco-a.pdf

6. Borrego AP, Ortueta ZTI, llisastigui AL, Domech HV, Pérez NG. Resorción dentaria interna. Presentación de un caso. Rev Haban Cienc Méd. 2010; 9 (2): 288-292.

7. León EPI. Estudio clínico del manejo endodóntico en caso de reabsorción Dentinaria Interna. Guayaquil, junio del 2013. Trabajo de graduación previo a la obtención del título de odontóloga. Disponible en: https:/l docplayer.es/amp//235 / 0858-Universidadde-guayaquil-facultad-piloto-de-odontologiatrabajo-de-graduacion-previo-a-la-obtencion-del-titulo-de-odontologa-tema-autor

8. Dusane SS, Raikwar KR, Bhagat BR, Suryavanshi RK. Rootless existence: A case report of internal resorption. J Int Clin Dent Res Organ. 2018; 10 (2): 97-100.
9. Irahola JGL, Maldonado VSP. Reabsorciones Dentinarias Internas relacionadas con tratamientos ortodónticos casos clínicos. Odontología actual. 2016; I (I): 8-I3.

10. Cadena Uyauri GM. "Tratamiento de la mancha en dientes anteriores". Guayaquil, Junio de 2013. Trabajo de graduación previo a la obtención del título de odontóloga. Disponible en: http://repositorio.ug.edu.ed/ handle/redug/3362

II. Kalender A, Öztan MD, Basmaci F, Aksoy $U$, Orhan K. CBCT Evaluation of multiple idiopathic internal resorptions in permanent molars. Case Report. BMC Oral Health 2014,14:39 Disponible en: http://www.biomedcentra.com//472-683///4/39

12. Peiris Barbieri, SV. Pérez Alfayate, R. González-Baquero A, Díaz Flores A, Valencia de Pablo, $\mathrm{O}$. Actualización sobre reabsorción dentinaria interna. Cient. Dent. 2012; 9 (3): 185-192.

13. Umashetty G, Hoshing U, Patil S, Ajgaonkar N. Management of Inflammatory Internal Root Resorption with Biodentine and Thermoplasticised Gutta-Percha. Case Rep Dent. 2015; article 452609: I-4. Available from: http://dx.doi.org/ / 0.I I55/2015/452609

14. Yaringaño-Medina N, Alamo-Palominol J, García-Rivera H. Tratamiento de reabsorción radicular interna perforante mediante uso de sustituto dentinario biocerámico y reconstrucción coronaria con resinas bulk fill: reporte de caso. KIRU. 20I7; I4 (2): I72-177.

15. Miranda LH, Lorenzo MR, Rodriguez SC. Implante transalveolar inmediato ante reabsorción dentinaria interna Rev Cubana Estomatol. 2019; 56 (I): 78-85. 
16. Lima TF, Gamba TO, Zaia AA, Soares AJ. Evaluation of cone beam computed tomography and periapical radiography in the diagnosis of root resorption. Aust Dent J. 2016; 61: 425-43I.

17. Kaiwar A, Ranjini M, Ashwini P, Pasha M, Meena N. Internal resorption managed by root canal treatment: Incorporation of CT with $3 \mathrm{D}$ reconstruction in diagnosis and monitoring of the disease. J Int Oral Health. 2010; 2 (I): 86-94.

18. Brun D, Scarparo R, Kopper P, Grecca F. Apical internal inflammatory root resorption and open apex treated with MTA: a case report. Revista Odonto Ciencia. 2010;25(2):213-2I5.

19. Li FC, Hung WC. Repair of a perforating internal resorption: two case reports. J Dent Sci. 2016; I I (3): 338-342.

20. Abdullah D, Hussein EF, Abd Ghani H. Management of Perforating Idiopathic Internal Root Resorption. Iran Endod J. 2017;12(2):257-260.

21. Madani Z, Moudi E, Bijani A, Mahmoudi E. Diagnostic Accuracy of Cone-Beam Computed Tomograpy and Periapical Radiograpy in Internal Root Resorption. IED. 2016; II (I): 5 I-6.

22. Kaval ME, Güneri P, Caliskan MK. Regenerative endodontic treatment of perforated internal root resorption: a case report. Int Endod J. 20।8;5I (I): I 28-37.

23. Yadav P, Rao Y, Jain A, Relhan N, Gupta S. Treatment of Internal Resorption with Mineral Trioxide Aggregates: A Case Repor. J Clin Diagn Res. 20 I3; I-7 (I0): 2400-240 I.
24. Magalhães Girelli CF, dos Santos AP, Marianelli MC, Santos Lacerda MFL, de Lima CO. The importance of cone beam computed tomography in the management of internal root resorption: a case report. Endodontic Practice Today. 20 |8; 12 (3): |9|-6.

25. Segovia RA, Sánchez LRT. Reabsorcion Dentinaria Interna. Reporte de un Caso. Rev ADM. 2005; LXII (2): 63-66.

26. Cordero $C$. Tomografía computadorizada: Una herramienta diagnóstica en endodoncia. Revista Odontología Vital. 20 I I; I (I4): 6-9.

27. Dalia A, Farah EH, Huwaina AG. Management of Perforating Idiopathic Internal Root Resorption. IEJ.2017; 12 (2): 257-60.

28. Suraj A, Gill GS, Saluja P, Setia Vikas.Management of A Rare Case of Communicating Internal-External Inflammatory Resorption. J Clin Diagn Res. 20I5; 9 (5): 39-40.

29. Araújo LCG de, Lins CV, Lima GA, Travassos RMC, Lins CC, dos Santos A. Study of Prevalence of Internal Resorption in Periapical Radiography of Anteriors Permanents Tooth. Int J Morphol. 2009;27( I):227-230.

30. Gupta V. Non- Surgical Repair of Internal Resorption with MTA: A case Report. Clinical Dentistry. 2017; II (6): 26-9.

31. Patni $P$, Jain $P$, Jain $S$, Hiremath $H$, Agarwal $P$, Patni M. Internal tunneling resoption associated with invasive cervical resorption. J Conserv Dent. 2018; 2 I (I): 105-8.

32. Ulusoy Öi, Paltun YN. Fracture resistance of roots with simulated internal resorption defects and obturated using different hybrid techniques. J Dent Sci. 20 I7; I2 (2): | 2 | - 25. 